\title{
Fast Legalization for Standard Cell Placement with Simultaneous Wirelength and Displacement Minimization
}

\author{
Tsung-Yi Ho and Sheng-Hung Liu \\ Department of Computer Science and Information Engineering \\ National Cheng Kung University
}

\begin{abstract}
Legalization is one of the most critical steps in modern placement designs. Since several objectives like wirelength, routability, or temperature are already optimized in global placement stage, the objective of legalization is not only to align the cells overlap-free to the rows, but also to preserve the solution of global placement, i.e., the displacement of cells needs to be minimized. However, minimizing displacement only is not enough for current timing-driven SoC designs. Blind displacement minimization may increase the half-perimeter wirelength (HPWL) of nets significantly that degrades the chip performance. In this paper, we propose a fast legalization algorithm for standard cell placement with simultaneous wirelength and displacement minimization. The main contributions of our work are: (1) a fast row selection technique by using k-medoid clustering approach; (2) an exact linear wirelength model to minimize both wirelength and total displacement; (3) a constant time approach to determine the median in trial placement stage. Compared with the state-of-the-art legalization algorithms, experimental results show that our legalizer acquires much better achievement in terms of HPWL, total and maximum displacements, and running time on legalized NTUplace3 global placement results on both ISPD 2005 and 2006 placement contest benchmarks.
\end{abstract}

Keywords: Displacement, Legalization, Placement, Wirelength.

\section{Introduction}

The conventional standard cell placement includes three stages: global placement, legalization, and detailed placement. Typically, global placement generates an initial placement with minimum total half-perimeter wirelength (HPWL) and tries to optimize some objectives such as routability, timing, temperature, and etc. This results in few cell overlap and the cells are not aligned to the rows. There has been several researches in the area of global placement in the past few years 3541116 18. After global placement stage, legalization targets (1) to remove overlaps between cell instances, (2) to put all instances on the rows in the core area, and (3) to minimize the displacements of instances between the initial placement and the legalized one. That is, legalization tries to make 
the placement legal and to preserve the result from the global placement stage. After legalization, detailed placement is used to improve the solution quality of the legal placement. Legalization with only displacement minimization may significantly induce the solutions with more HPWL than global placement stage. For example, if cell $a$ is to be legalized in Figure 1 (a), blind legalization with only displacement minimization may increase the HPWL as shown in Figure 1 (b). However, by taking HPWL into consideration, we can legalize cell $a$ with both displacement and HPWL minimization.

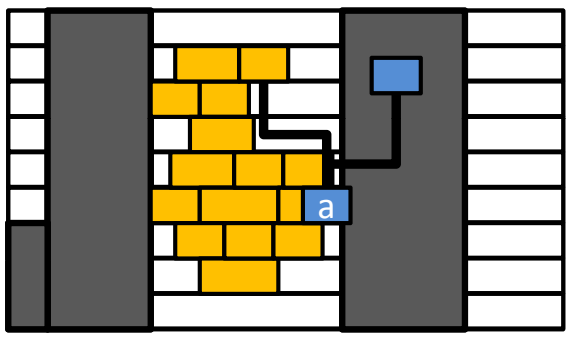

(a) Original

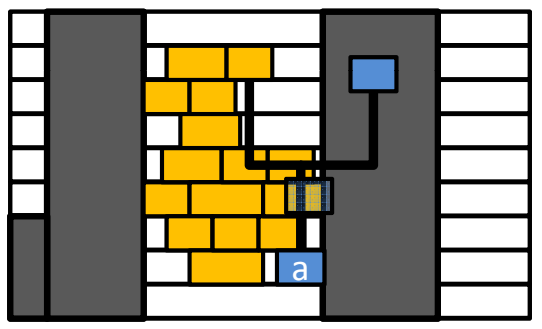

(b) Larger HPWL
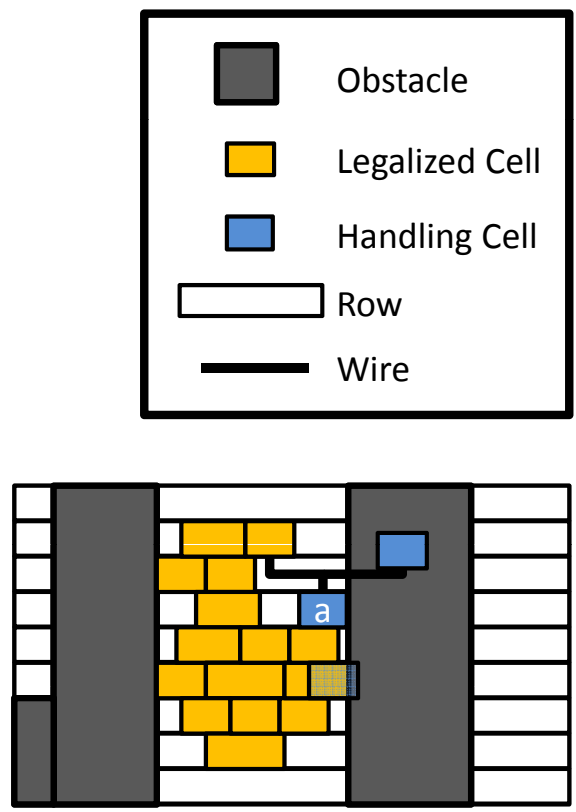

(c) Smaller HPWL

Fig. 1. (a) Original placement before legalizing cell $a$. (b) Placement with larger HPWL after legalizing cell $a$. (c) Placement with smaller HPWL after legalizing cell $a$.

\subsection{Previous Work}

Existing legalization techniques include network flow [26], ripple cell movement [8, dynamic programming [1], simulated annealing [17, single row optimization 10, diffusion-based method [15, computational geometry [13, packing [7], and quadratic programming [18]. Domino 6] partitions cells in subcells (all having the same height and width) and rows in places, and assigns them by using a min-cost-max-flow approach. Similar to Domino, [2 assigns sets of modules to row regions. Mongrel [8] uses a greedy heuristic to move cells from overflowed bins to under capacity bins in a ripple fashion based on total wirelength gain. Fractional Cut [1] assigned cells to rows by dynamic programming 
and the cells of each row are packed from left to right. Sarrafzadeh [17] uses simulated annealing for legalization. The authors of [10] assigned cells to the rows by cell juggling and the cells of each row are placed by finding a shortest path in a graph. Diffusion-based placement migration is presented in [15] to remove cell overlap incrementally. In [13], cells are spread and aligned to rows by computational geometry techniques. Sarrafzadeh [17] uses a cell shifting technique to reduce the maximum bin density. HPWL-driven legalizers only target on HPWL minimization within a row by dynamic programming approach 910.

Tetris is a fast greedy heuristic which is widely used in industry 7]. Tetris sorts the cells first, and legalizes one cell at a time then. The legalization of one cell is done by moving the cell over the rows, and within a row by moving the cell over free sites. This movement is done until the best free site is found. Once a cell has been legalized, it will not be moved anymore. This results in a high total cell displacement during legalization. Recently, Spindler et al. present a similar approach, called Abacus [19, that it places a cell from row to row until the location with the smallest displacement is found. However, as a cell is placed into a row, the legalized cells in that row are re-placed by dynamic programming technique to minimize the total displacement. Both Tetris and Abacus scan rows with minimum cost vertically. Tetris scans all rows (see Figure 2 (a)). Abacus sorts the rows by the y-position. A lower bound of the cost is computed by assuming cells are only moved vertically. Abacus moves the cell to the best row first, and then tries to move the cell to the rows with the lower bound not exceeding the minimal cost of an already found legal position (see Figure 2 (b)). In this paper, our legalizer constructs the binary index tree for the rows via clustering techniques. By traversing the binary index tree, our legalizer only scans some candidate rows then the running time is significantly reduced (see Figure $2(\mathrm{c}))$.

\subsection{Our Contribution}

The main contributions of our work are: (1) a fast row selection technique by using k-medoid clustering approach; (2) an exact linear wirelength model to minimize both total displacement and HPWL; (3) a constant time approach to determine the median in trial placement stage. The remainder of this paper is organized as follows. Section 2 details our algorithm. Section 3 shows the experimental results. Finally, concluding remarks are given in Section 4.

\section{Algorithm}

Figure 3 shows the overview of our legalization algorithm. First, a binary index tree for rows is constructed for fast row scanning (line 1). After that, cells are sorted according to the sum of their x-position and the half of their width (line 2 ). Then, cells are legalized one by one according to the sorting order (line 3-8). "DFS" (line 5) adopt depth-first search manner to search the best row for each 


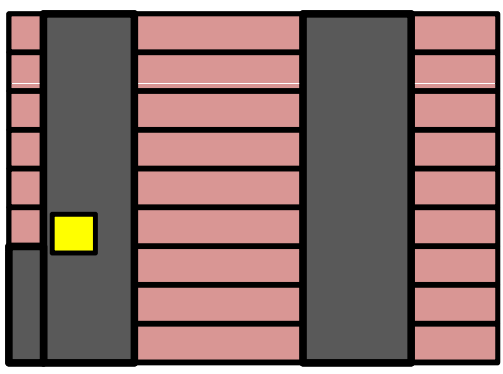

(a) Tetris

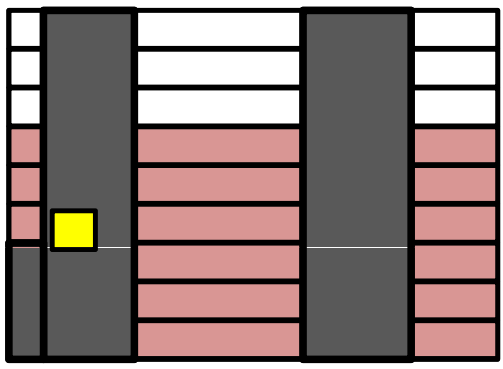

(b) Abacus
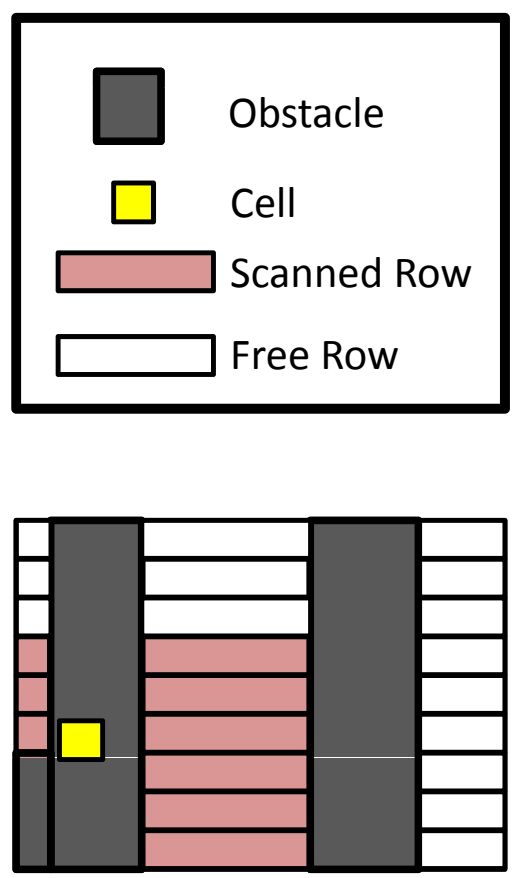

(c) Ours

Fig. 2. (a) Tetris scans all rows. (b) Abacus scans the rows by y-position. (c) Our legalizer only scans the rows when they are necessary.

cell; i.e., the cost (the sum of HPWL and displacement of the cell) of the row is minimum by using the binary index tree for row scanning (line 1). Then the cell is inserted to the best row for HPWL and displacement minimization (line 6).

\subsection{Binary Index Tree}

Fast row scanning can be achieved by searching a binary index tree constructed by top-down row clustering technique. In this paper, we adopt k-medoid [12 clustering algorithm as the main method for clustering rows. Typically, k-medoid algorithm clusters items by using their similarity. To cluster rows, we define the similarity between rows as the distance between each row. If the distance between each row is far, the similarity is small, and vice versa. Initially, all rows are in a cluster, called root cluster. Then all rows are clustered into two clusters by using $\mathrm{k}$-medoid algorithm $(\mathrm{k}=2)$ where the centers are central rows of row clusters. Iteration of clustering terminates until the number of rows in every cluster is less than 3. Finally, we construct a binary index tree for row clusters. The relation between rows and binary index tree is shown in Figure 4. 


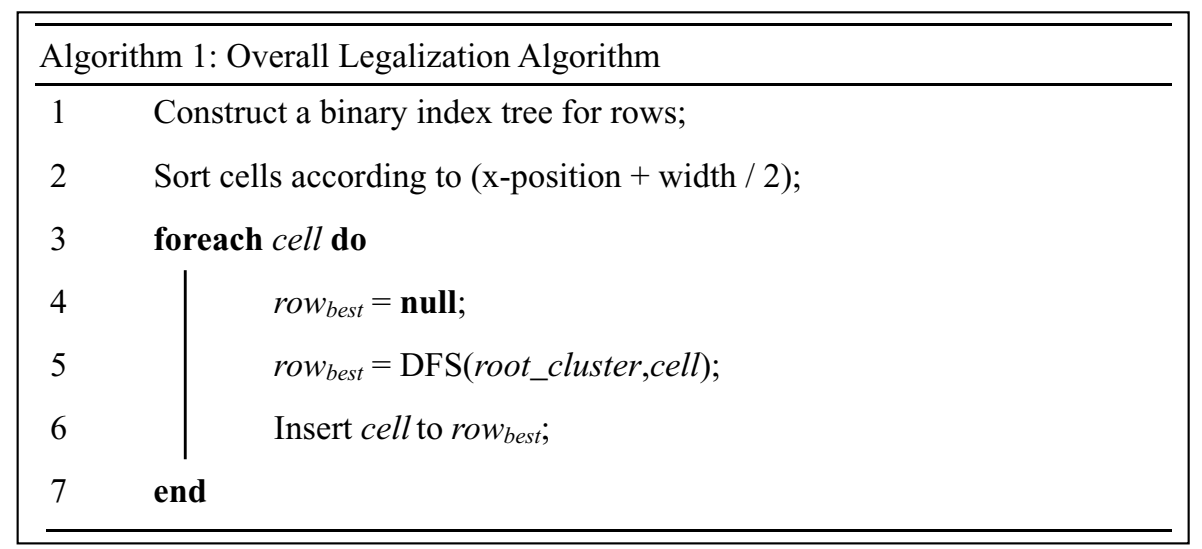

Fig. 3. Overall algorithm of our legalizer
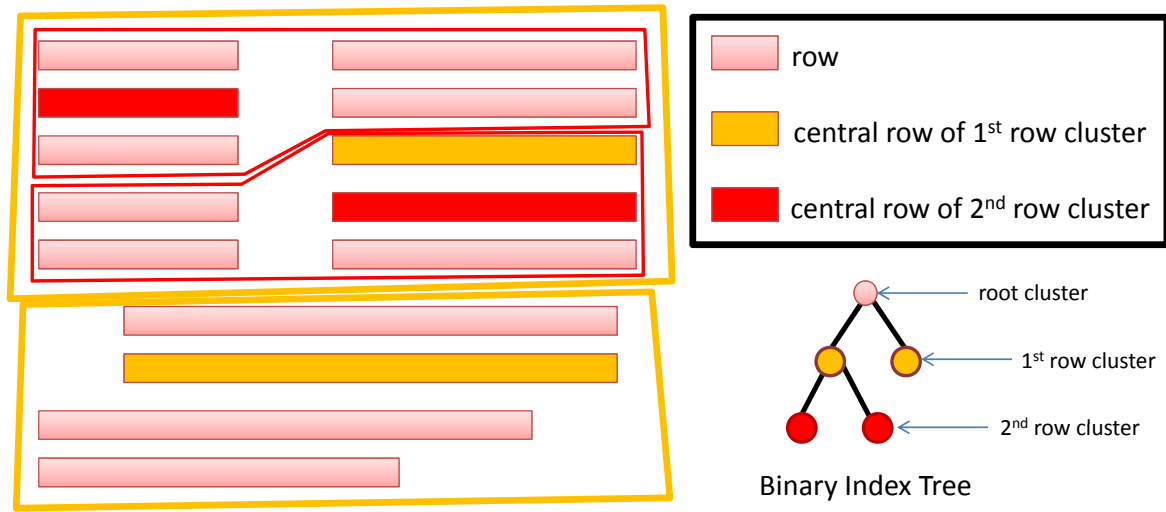

Binary Index Tree

Fig. 4. Top-down clustering constructs a binary index tree for rows

\subsection{Row Scanning}

Before placing cells into the rows, legalizers perform row scanning then do trial placement for cells. Different from previous legalizers that scan all rows, our legalizer can find the best row efficiently by searching on the binary index tree. As mentioned earlier, the nodes in the binary index tree represent row clusters. The edge cost in the binary index tree is the distance from a cell to the row cluster. To compute the distance from a cell to the row cluster, we need to compute the distance between rows first. $D_{\min }\left(\right.$ row $_{i}$, row $\left._{j}\right)$ and $D_{\max }\left(\right.$ row $_{i}$, row $\left._{j}\right)$ are two kinds of the distance from row $r_{i}$ to $r_{0} w_{j}$. We adopt $D_{\min }\left(r o w_{i}, r o w_{j}\right)$ as the distance for clustering rows by using k-medoid algorithm and $D_{\max }\left(\right.$ row $_{i}$, row $\left._{j}\right)$ as the distance used for computing the edge cost of the binary index tree. If

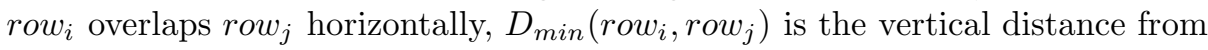


row $_{i}$ to row $_{j}$ (see Figure 5 (a)). If row does not overlap row $_{j}$ horizontally, $D_{\min }\left(\right.$ row $_{i}$, row $\left._{j}\right)$ is the sum of vertical and horizontal distance from row to $_{i}$ row $_{j}$ (see Figure $\left.5(\mathrm{~b})\right)$. $D_{\max }\left(\operatorname{row}_{i}, r \mathrm{w}_{j}\right)$ is the sum of the vertical distance and maximum horizontal distance from the left of $r_{0} w_{i}$ to the left (see Figure 5 (c)) of row $_{j}$ or from the right of row to the right of row $_{j}$ (see Figure $5(\mathrm{~d})$ ). Moreover, the computation of distance from cells to row clusters is the same with distance between rows.
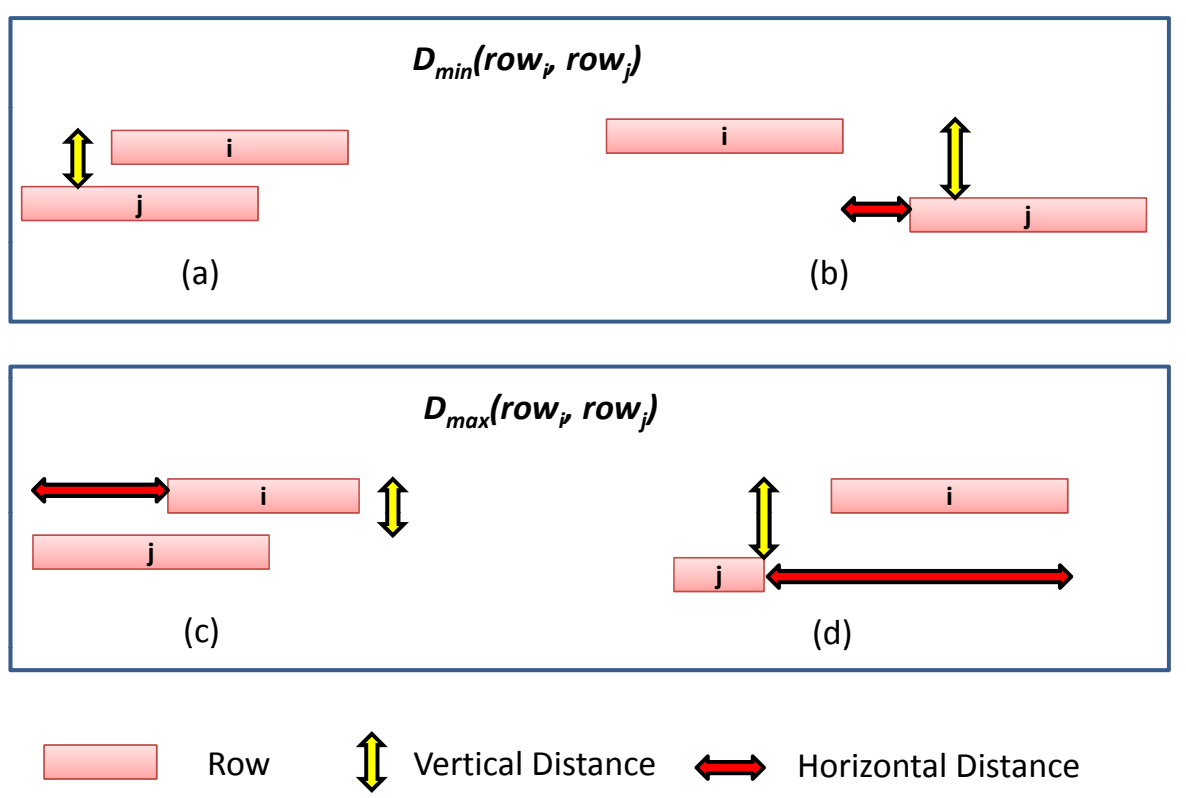

Fig. 5. (a) $D_{\min }\left(\right.$ row $_{i}$, row $\left._{j}\right)$ with horizontal overlaps. (b) $D_{\min }\left(\right.$ row $_{i}$, row $\left._{j}\right)$ without horizontal overlaps. (c) $D_{\max }\left(\right.$ row $\left._{i}, r_{\mathrm{r}} \mathrm{w}_{j}\right)$ with horizontal overlaps. (d) $D_{\max }\left(\right.$ row $_{i}$, row $\left._{j}\right)$ without horizontal overlaps.

After the distance between rows is computed, we defined radius of a row cluster is the farthest distance between its central row to other rows. According to triangle inequality, the distance from a cell to the row cluster, i.e. $D_{\text {min }}($ cell,row_cluster), can be derived as shown in Figure 6 .

A depth-first search (DFS) approach is used for finding the best row. Algorithm 2 describes the function DFS. DFS starts from root cluster. The cell is always moved into the nearer cluster for trial placement first (line 5-18). The cell is not moved to cluster if $D_{\min }($ cell, row_cluster) is larger than the current best displacement. The cost of the row is the sum of both HPWL and displacement of the cell after the cell is moved to the row (line 21). After DFS, the best row can be found quickly, then cells will be inserted into it. For simultaneous wirelength and displacement minimization, the cost of row for placing a cell depends on the displacement and the HPWL of it. Since a cell may be connected by multiple 


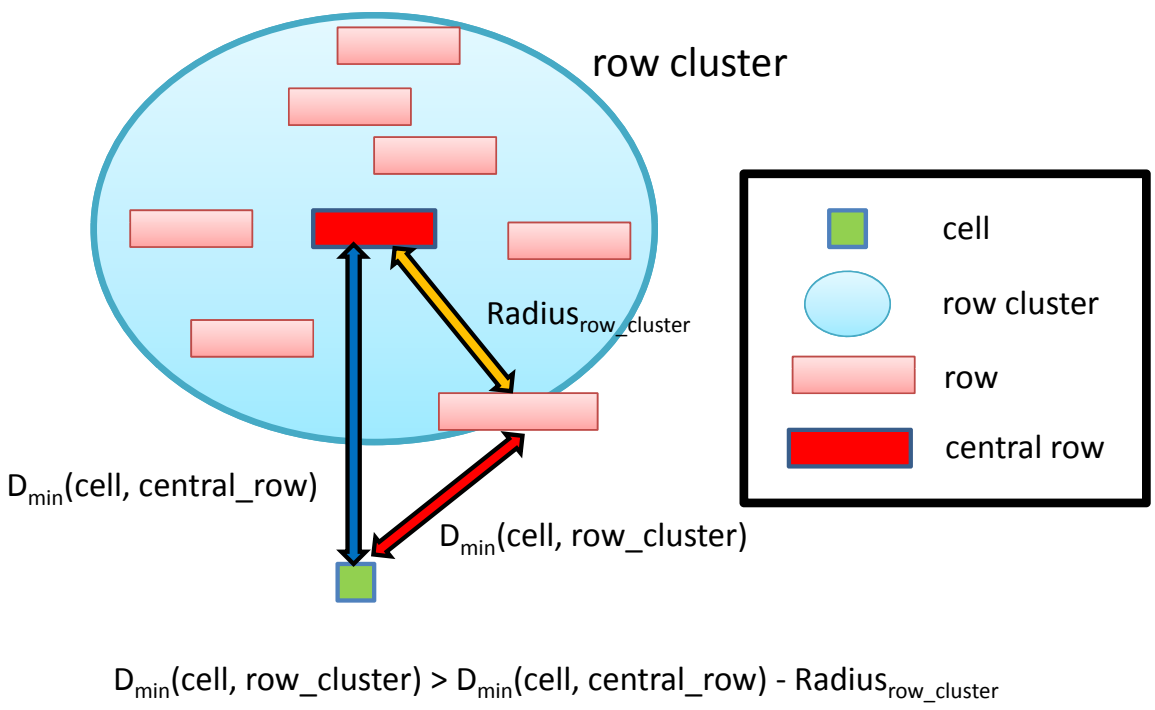

Fig. 6. Illustration of the minimum distance computation between a cell and a row_cluster

nets, the cost by simply adding the displacement and the HPWL of a cell may not be precise. Furthermore, we not only target to minimize total displacement but also to minimize maximum displacement, we consider both factors by a combined cost function. The cost function is defined as follows:

$$
\operatorname{Cost}(i)=H P W L+N_{i}\left(\alpha \cdot\left(D P_{S}\right)+\beta \cdot\left(D P_{P}\right)\right)
$$

where $N_{i}$ represents the number of the net belongs to cell $i . D P_{S}$ is the cell displacement between original position and legalized position. $D P_{P}$ is the total increased displacement caused by inserting one cell into row. $\alpha, \beta$ are userspecified parameters. An example of cost computation is shown in Figure 8.

\subsection{Obstacle-Aware Cell Ordering}

Modern chip designs often consist of many preplaced blocks, such as analog blocks, memory blocks, and/or I/O buffers, which are fixed in the chip and cannot overlap with other blocks. These preplaced blocks, i.e., obstacles, impose more constraints on the legalization problem. A legalization algorithm without considering obstacles may significantly induce increasing cell displacement or inferior solutions. Different from all previous works that insert cell according to its $\mathrm{x}$-position in the global placement, we insert cell according to its central position (i.e., x-position of cells + cell width/2). As illustrated in Figure 9, an obstacle lies in the middle of the row shred the row into two subrows. If cells 


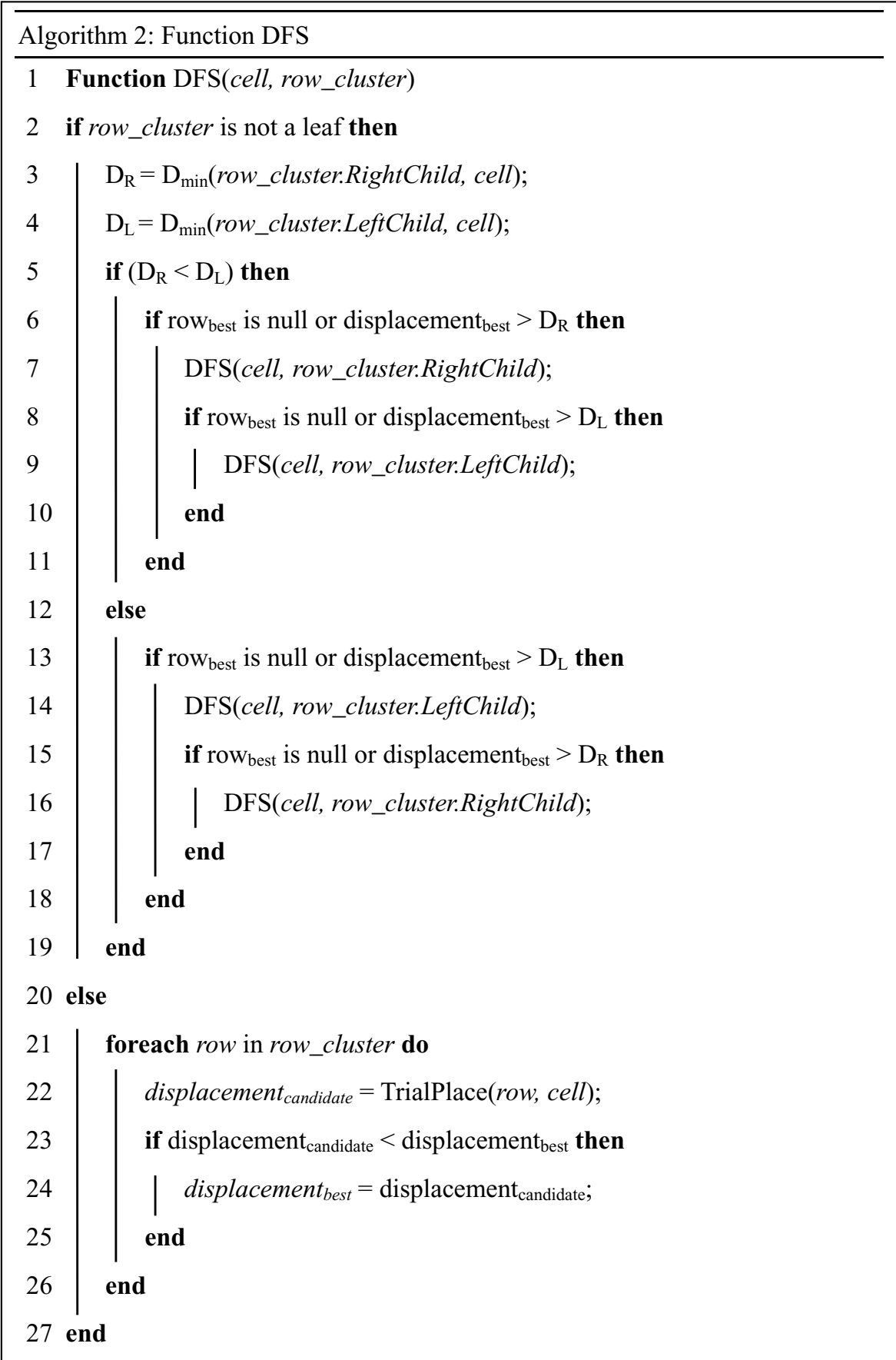

Fig. 7. The algorithm of function DFS 
are inserted by x-position, the larger cell 1 will be inserted first but cause larger displacement for cell 2 and 3 since they are inserted to another subrow that across the obstacle (See Figure 9 (a)). It is not worth because only cell 1 is inserted into the left subrow with smaller displacement and cell 2 and 3 are squeezed to right subrows with larger displacement. If cells are inserted by their central position, most of the smaller cells that are capable of being inserted to fragmentary subrows can reduce the total displacement significantly (See Figure 9 (b)). Moreover, it is capable of handling the relative order problem.
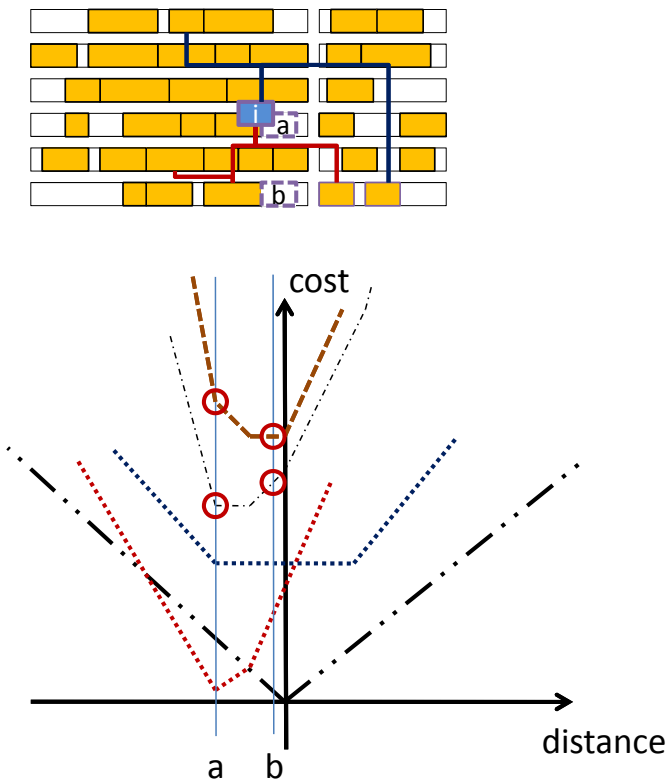

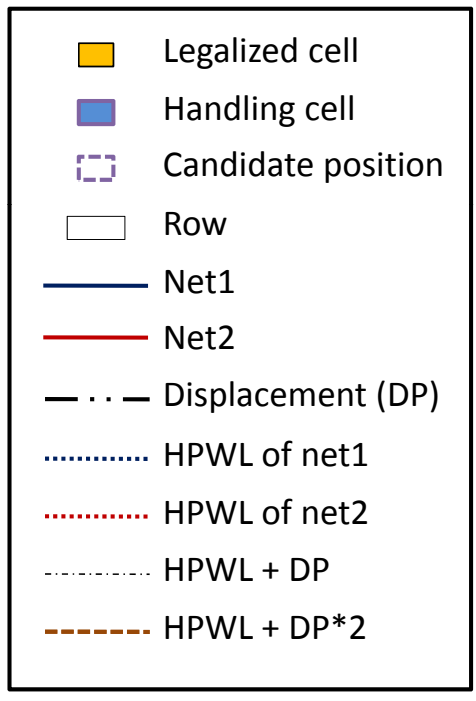

Fig. 8. Illustration of the cost(i) to find the best row

\subsection{Cell Insertion}

Most of previous works use simple quadratic wirelength model to measure cell displacement. The drawback lies in the preciseness since the square of larger cell displacement will have dramatically difference. Different from Abacus that uses quadratic model, we adopt an exact linear wirelength model for measuring cell displacement. Cells of one row are abutting in the legal placement and form a cluster. Assume the row has $c$ clusters and cluster $_{c}$ has $n$ cells which are sorted by their global x-position + width $/ 2$. To minimize the cell displacement, the objective function is defined as follows:

$$
\min \sum_{i=1}^{n}\left|x_{i}^{c}-x_{i}^{\prime c}\right|
$$



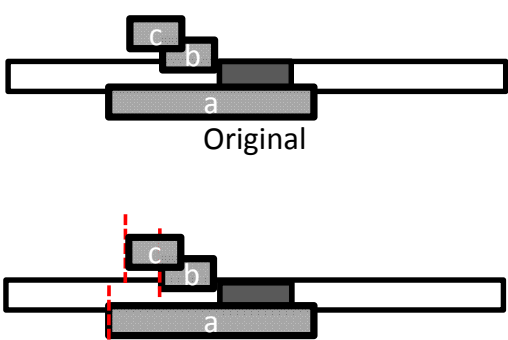

(a) Scan by left. Order is $a>c>b$.

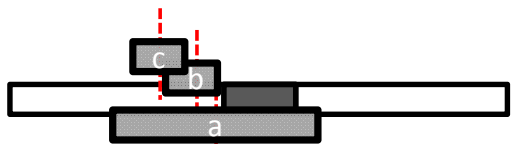

(b) Scan by center. Order is $c>b>a$.
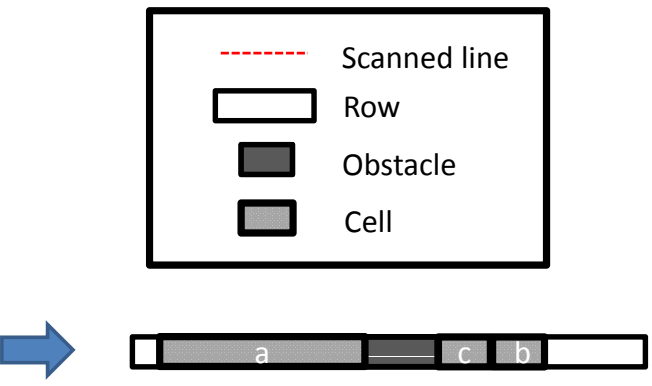

Total displacement $\uparrow$
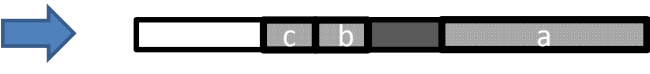

Total displacement $\downarrow$

Fig. 9. Ordering for obstacle-aware cell insertion

where $x_{i}^{\prime c}$ and $x_{i}^{c}$ are initial and legalized position of cluster $c$. The constraint (2) assures that there is no overlap between the cells and is defined as follows:

$$
x_{i}^{c}-x_{i-1}^{c} \geq w_{i-1}^{c} \quad i=2, \ldots, n
$$

where $w_{i}^{c}$ is cell width. However, solving linear programs with " $\geq$ " constraints is time consuming in general. If the same solution of the linear program is found by "=" constraints, then the linear program is solved quite fast by solving one linear equation. The situation that "=" constraints are sufficient is given if all cells of one row are abutting in the legal placement and form a cluster. There, two cells are "abutting" if there is no free space between them in the legal placement. With only "=" constraints, (2) is transformed to:

$$
x_{i}^{c}=x_{1}^{c}+\sum_{k=1}^{i-1} w_{k}^{c} \quad i=2, \ldots, n
$$

By (3), we can transform (1) into (4) such that the optimal value only depends on variable $x_{1}^{c}$.

$$
\min \sum_{i=1}^{n}|x_{1}^{c}-\underbrace{\left[x_{i}^{c}-\sum_{k=1}^{i-1} w_{k}^{c}\right]}_{d_{i}^{c}}|
$$

where $d_{i}^{c}$ is the candidate position of cluster $c$ for cell $i$ and all $d_{i}^{c}$ form a set $D_{c}$. The optimal $x_{1}^{c}$ can be derived by finding the median $m$ of all $d_{i}^{c}$. 


\subsection{Find Median}

As shown in Figure 10, a row has several clusters. The positions of these clusters are determined by $X_{c}$. If the clusters are overlapped, they will be merged and form a new cluster.

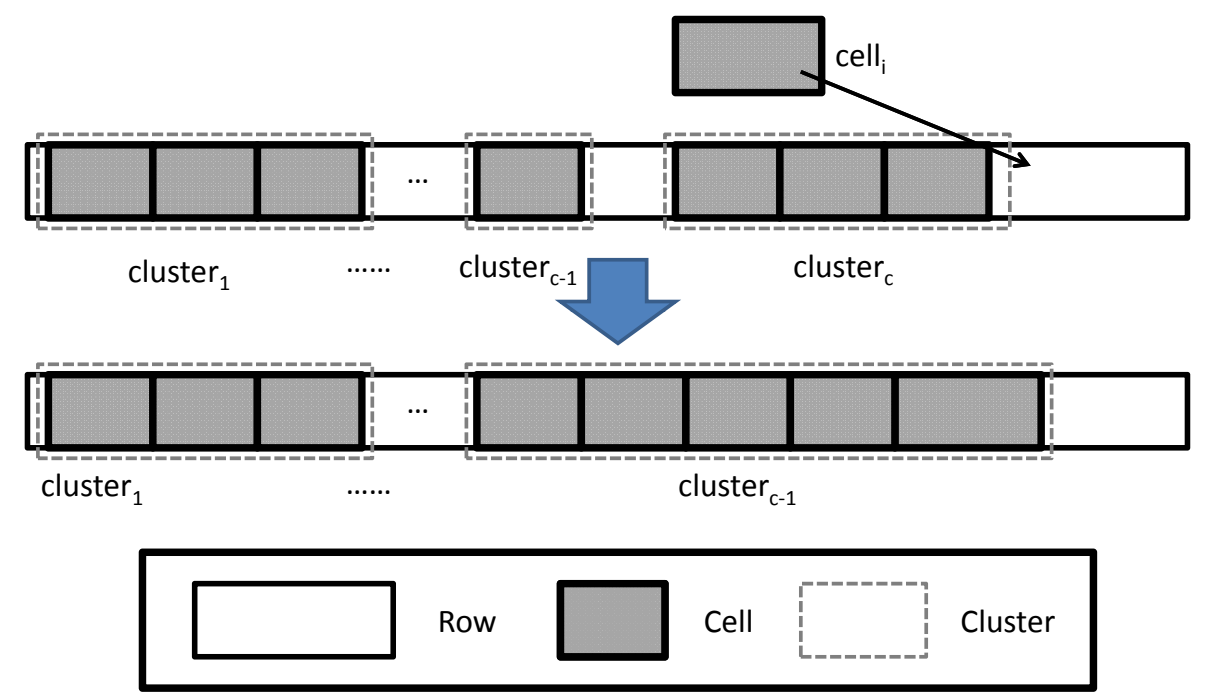

Fig. 10. Illustration of cluster merging

The intuitive method for finding the median is to sort $n$ elements in $D_{c}$ while the time complexity of sorting for trial placement and insertion are both $O(n \log n)$. An efficient method for finding the median is to apply the deterministic partitioning algorithm from quicksort while the time complexity for trial placement and insertion are both $O(n)$. In this paper, we adopt red-black trees for finding the median while the time complexity for trial placement and insertion are both $O(\log n)$. Moreover, red-black trees can be used to record already sorted $D_{c}$ since $D_{c}$ will not be changed after cells are placed. Thus, we can derive the set of $X_{c}$ as follows:

$$
X_{c}=\left\{x_{c}(i)\right\} \quad i=2, \ldots, n
$$

where $x_{c}(i)$ corresponds to the already sorted elements in $D_{c}$.

Since cells are not really placed on rows in trial placement stage, unnecessary movement of cells can be pruned in this stage. By exploring all possible movements of clusters, we found only the rightmost cluster (i.e., cluster $_{c}$ ) will move to the left and others will stay still. By this observation, we have 3 lemmas for our trial placement stage. By these 3 lemmas, the time complexity of our trial 
placement is only $\mathrm{O}(1)$ since we only need to compare 3 possible positions then we can find the best position for all clusters. The comparison of time complexity in listed in Table 1.

Table 1. Time complexity for trial placement and cell insertion

\begin{tabular}{|c|c|c|}
\hline & Trial & Insert \\
\hline Sorting algorithm & $\mathrm{O}(\mathrm{n} \log n)$ & $\mathrm{O}(\mathrm{nlog} n)$ \\
\hline SELECT algorithm & $\mathrm{O}(\mathrm{n})$ & $\mathrm{O}(\mathrm{n})$ \\
\hline Red-Black tree & $\mathrm{O}(\log n)$ & $\mathrm{O}(\log n)$ \\
\hline Ours & $\mathrm{O}(1)$ & $\mathrm{O}(\log n)$ \\
\hline
\end{tabular}

Lemma 1. If the number of the cells in cluster $_{c}$ is even, the cell $i$ is placed to the right of cluster ${ }_{c}$ and the position of cluster ${ }_{c}$ will not be changed.

Proof: Let $c$ is the number of clusters in a row. $N_{c}$ is the number of the cells in cluster $_{c}$. $W_{c}$ is the width of cluster $_{c}$. The candidate position for cell $i$ is $\overline{x_{i}}$ which is the difference of $W_{c}$ and the original x position of cell $i$. If cell $i$ and cluster $_{c}$ overlap horizontally, $\overline{x_{i}}$ is less than the original x position of cluster $_{c}$.

As shown in Figure 11, assume $N_{c}$ is an even number. The original x position of cluster $_{c}$ is the median of $X_{c}$ (i.e., $\frac{N_{c}}{2}$ ). Therefore, there are $\frac{N_{c}}{2}-1$ elements that are smaller than the original $\mathrm{x}$ position of cluster $_{c}$, and $\frac{N_{c}}{2}$ elements that are larger than the original x position of cluster $_{c}$. After cell $i$ and cluster $_{c}$ are merged, $\overline{x_{i}}$ is added into $X_{c}$. Since $\overline{x_{i}}$ is smaller than the $\mathrm{x}$ position of cluster $c$, the number of $X_{c}$ which smaller than the original x position of cluster $\mathrm{c}$ is $\frac{N_{c}}{2}$. Therefore, the median of $X_{c}$ does not changed. The new x position of cluster $_{c}$ is the original $\mathrm{x}$ position of cluster $_{c}$.

Lemma 2. If the number of the cells in cluster $_{c}$ is odd, the cell $i$ is placed to the right of cluster ${ }_{c}$ and the position of cluster ${ }_{c}$ will shift to the left.

Proof: As shown in Figure 12, assume $N_{c}$ is a odd number and the original $\mathrm{x}$ position of cluster $_{c}$ is the median of $X_{c}$ (i.e., $\frac{N_{c}+1}{2}$ ). Therefore, the number of $X_{c}$ which smaller than the original $x$ position of cluster is $\frac{N_{c}+1}{2}-1$. The number of $X_{c}$ which larger than the original x position of cluster $_{c}$ is $\frac{N_{c}+1}{2}-1$. After cell $i$ and cluster $_{c}$ are merged, $\overline{x_{i}}$ is added into $X_{c}$. Since $\overline{x_{i}}$ is smaller than the original x position of cluster $_{c}$, the number of $X_{c}$ which smaller than the original $\mathrm{x}$ position of cluster $_{c}$ is $\frac{N_{c}+1}{2}$. Therefore, the median of $X_{c}$ will be changed. The new x position of cluster $_{c}$ is $\frac{N_{c}+1}{2}$ of new $X_{c}$ instead of the original x position of cluster $_{c}$. In other words, new x position of cluster $_{c}$ is the maximum one between $\left(\frac{N_{c}+1}{2}-1\right)$ of original $X_{c}$ and $\left.\overline{x_{i}}\right)$.

By Lemma 2, cluster $_{c}$ may overlap with cluster $_{c-1}$ when cluster $_{c}$ shifts to the left. In this situation, cluster $_{c-1}$ and cluster $_{c}$ are merged and then form a new and bigger cluster $_{c-1}$ and the number of the clusters of the row becomes $c-1$. 


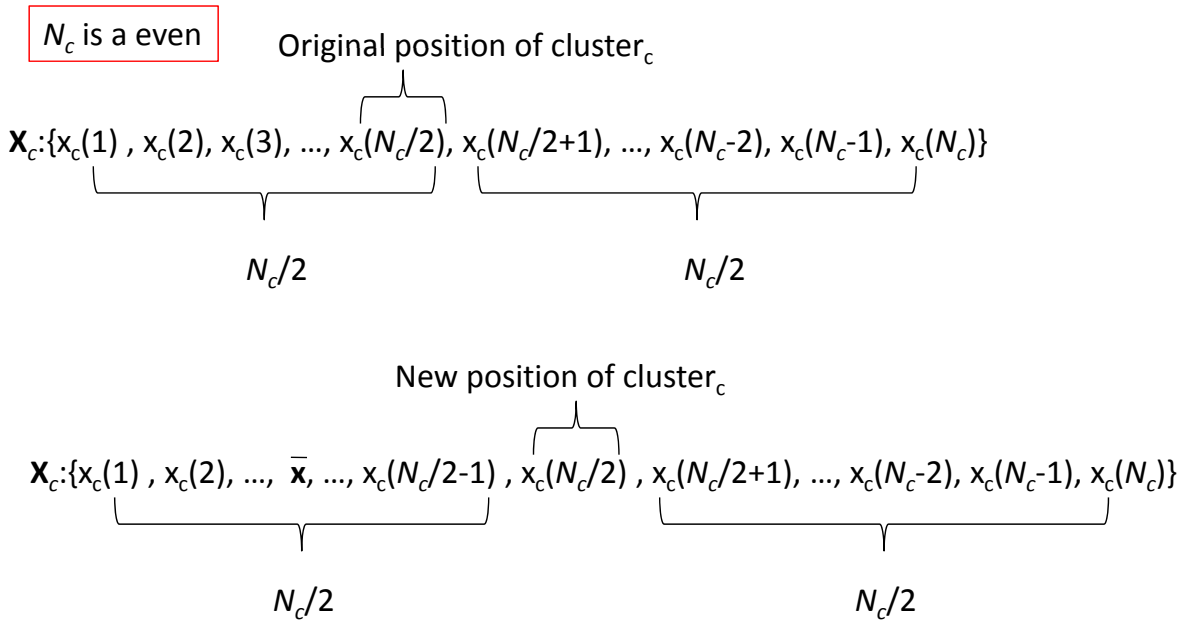

Fig. 11. $N_{c}$ is an even number
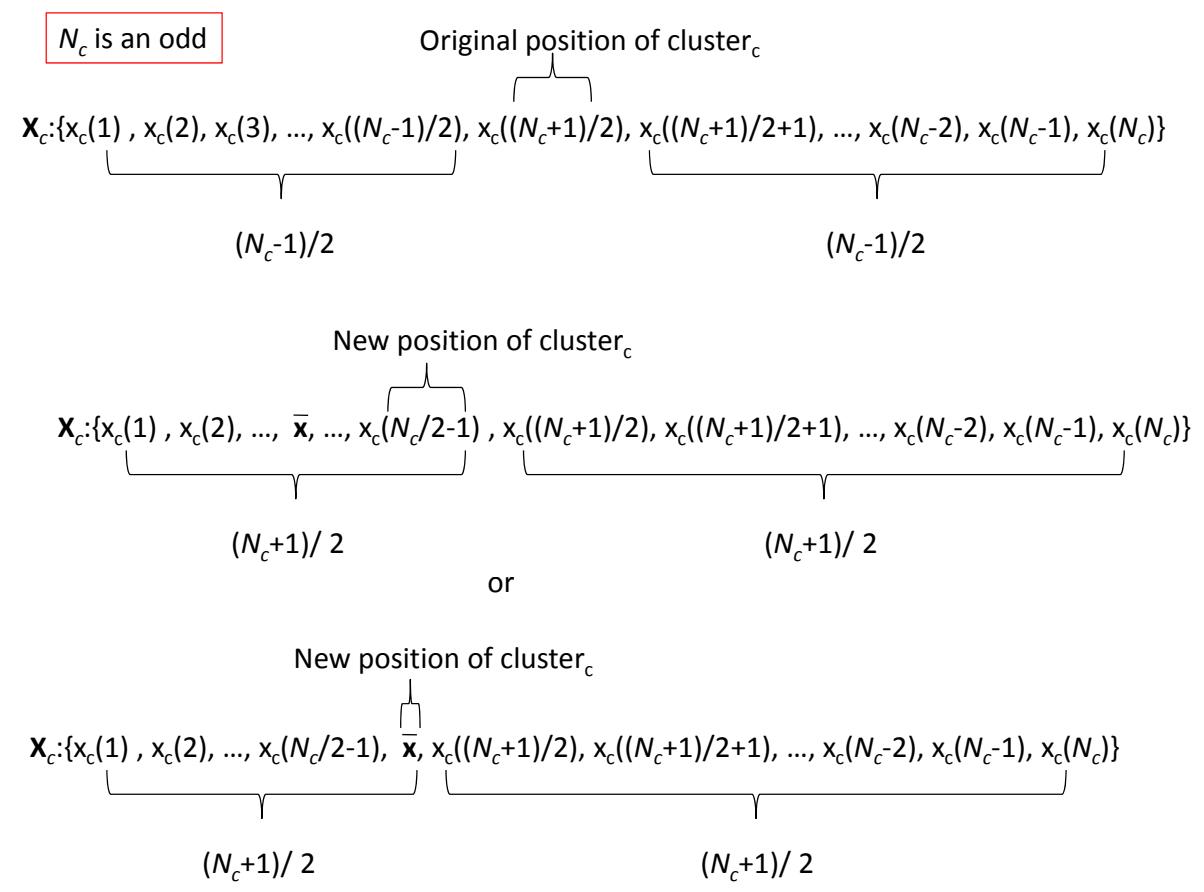

Fig. 12. $N_{c}$ is an odd number

Under this circumstance, we further observe that although cluster $_{c}$ shifts to the left, the positions of other $c-1$ clusters will not be influenced by cluster ${ }_{c}$. The details are described in Lemma 3. 

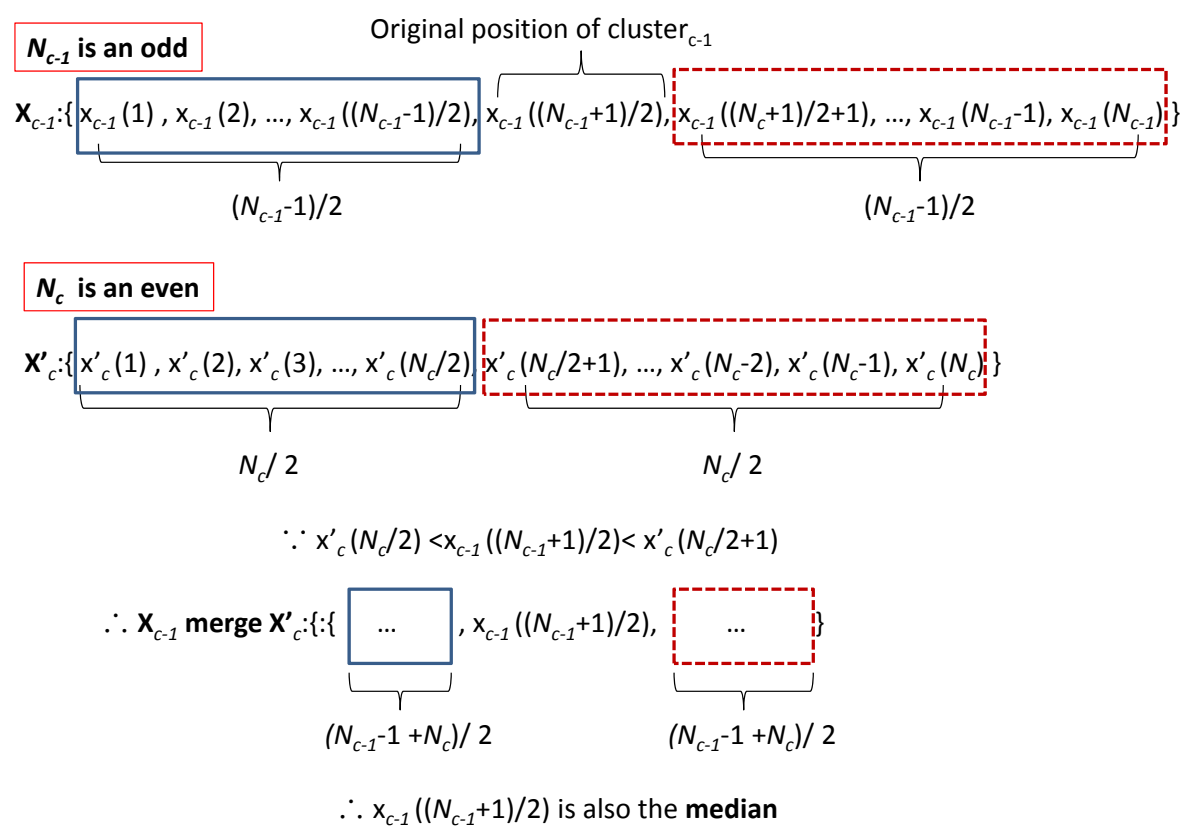

Fig. 13. Merge process of cluster $_{c-1}$

Lemma 3. cluster $_{c}$ has no influence on other $n-1$ clusters.

Proof: As shown in Figure 13, $X_{c}^{\prime}$ is the difference between $X_{c}$ and $W_{c-1}$. If a new cell added to the row, it will cause cluster $_{c}$ overlapped with cluster $_{c-1}$, then cluster $_{c-1}$ and cluster $_{c}$ will be merged together. After cluster $_{c-1}$ and cluster $_{c}$ are merged, the position of the new cluster $_{c-1}$ is found by the median of new $X_{c-1}$ (i.e., original $X_{c-1}$ merged with $X_{c}^{\prime}$ ). The median of original $X_{c}^{\prime}$ (i.e., $\left.X_{c}^{\prime}\left(N_{c} / 2+1\right)\right)$ is larger than the median of $X_{c-1}$ (i.e., $\left.X_{c-1}\left(\left(N_{c-1}+1\right) / 2\right)\right)$, because cluster $_{c-1}$ and original cluster $_{c}$ are not overlapped. Once a new cell added to the row, it will cause cluster $_{c}$ overlaps cluster $_{c-1}$, the median of new $X_{c}^{\prime}$ (i.e., $\left.X_{c}^{\prime}\left(N_{c} / 2\right)\right)$ will be smaller than the median of $X_{c-1}$ (i.e., $X_{c-1}\left(\left(N_{c-1}+\right.\right.$ $1) / 2)$ ). Therefore, after cluster $_{c-1}$ and cluster $_{c}$ are merged, the median of new $X_{c-1}$ is unchanged (i.e., $\left.X_{c-1}\left(N_{c-1}+1\right) / 2\right)$. It means that the x position of new cluster $_{c-1}$ is unchanged, and the position of cell is the sum of $\left(X_{c-1}\left(N_{c-1}+\right.\right.$ 1)/2), $W_{c-1}$, and $L_{c}$.

In trial place step, the position of the cell on the row can be calculated by finding the maximum of the following three numbers: $x^{\prime}$, the sum of the $\left.\frac{N_{c}+1}{2}-1\right)$ of $X_{c}$ and $W_{c}$, and the sum of the x position of cluster $_{c-1}$ (i.e., $\left(X_{c-1}\left(\left(N_{c-1}+\right.\right.\right.$ $1) / 2))), W_{c-1}$, and $L_{c}$. 


\section{Experimental Results}

We have implemented our legalizer in the $\mathrm{C}++$ language on a 2-GHz 64-bit Linux machine with 16GB memory. For fair comparison, we evaluate NTUplace3 [5], Tetris [7, Abacus [19], and Kahng [9] on the same platform. Furthermore, to verify the efficiency and effectiveness of our legalizer, we perform experiments on two benchmark suites, such as ISPD 2005 and 2006 placement contest benchmarks [22]. The global placement results are obtained from NTUplace3 [5]. Extensive experiments demonstrate that in terms of HPWL, total and maximum displacements, and running time, we acquire much better achievement than all the state-of-the-art algorithms such as Tetris [7, Abacus [19], and Kahng 9] in any aspect.

\subsection{Benchmarks}

The cell numbers of the ISPD 2005 benchmarks range from $210 \mathrm{~K}$ to $2169 \mathrm{~K}$, the fixed macro numbers range from 543 to 23084, and the row numbers range from 890 to 2694 . The cell numbers of the ISPD 2006 benchmarks range from $330 \mathrm{~K}$ to $2507 \mathrm{~K}$, the fixed macro numbers range from 337 to 26582 , and the row numbers range from 930 to 4182 . The benchmark information is listed in Table 2.

Table 2. Statistics of two benchmark suites of the ISPD placement contest

\begin{tabular}{|c|c|c|c|c|c|c|c|}
\hline \multicolumn{4}{|c|}{ ISPD 2005 Benchmarks } & \multicolumn{4}{c|}{ ISPD 2006 Benchmarks } \\
\hline Name & \# Cells & \# Fixed Macros & \# Rows & Name & \# Cells & \# Fixed Macros & \# Rows \\
\hline adaptec1 & 210904 & 543 & 890 & adaptec5 & 843128 & 646 & 1944 \\
\hline adaptec2 & 254457 & 566 & 1170 & newblue1 & 330073 & 337 & 930 \\
\hline adaptec3 & 450927 & 723 & 1944 & newblue2 & 441516 & 1277 & 1925 \\
\hline adaptec4 & 494716 & 1329 & 1944 & newblue4 & 646139 & 3422 & 1524 \\
\hline bigblue1 & 277604 & 560 & 890 & newblue5 & 1233058 & 4881 & 2130 \\
\hline bigblue2 & 534782 & 23084 & 1566 & newblue6 & 1255039 & 6889 & 2316 \\
\hline bigblue3 & 1093034 & 1293 & 2316 & newblue7 & 2507954 & 26582 & 3258 \\
\hline bigblue4 & 2169183 & 8170 & 2694 & & & & \\
\end{tabular}

\subsection{HPWL}

In the first experiment, we evaluate the HPWL of our legalizer on the both benchmarks. On legalized NTUplace3 [5] global placements on ISPD 2005 placement contest benchmarks, the HPWL is $1.92 \mathrm{X}, 1.19 \mathrm{X}$ and $1.92 \mathrm{X}$ of our legalizer compared to Tetris 7, Abacus [19, and Kahng 9], respectively. It should be noted that Kahng [9] only focusing on HPWL minimization. Moreover, we also compare the HPWL after our legalizer with the original HPWL of NTUplace3 global placement results [5, we can further reduce HPWL by $18 \%$. The details of HPWL experiments on ISPD 2005 benchmarks are listed in Table 3. 
Table 3. Wirelength comparison between NTUplace3, Tetris, Abacus, Kahng, and ours on ISPD 2005 benchmarks

\begin{tabular}{|c|c|c|c|c|c|}
\hline ISPD 2005 Benchmarks & NTUplace3 [5] & Tetris [7] & Abacus [19] & Kahng [9] & Ours \\
\hline Name & HPWL & HPWL & HPWL & HPWL & HPWL \\
\hline adaptec1 & $9.56 \mathrm{E}+07$ & $1.20 \mathrm{E}+08$ & $9.50 \mathrm{E}+07$ & $1.19 \mathrm{E}+08$ & $8.00 \mathrm{E}+07$ \\
\hline adaptec2 & $9.98 \mathrm{E}+07$ & $1.39 \mathrm{E}+08$ & $1.01 \mathrm{E}+08$ & $1.39 \mathrm{E}+08$ & $8.80 \mathrm{E}+07$ \\
\hline adaptec3 & $2.43 \mathrm{E}+08$ & $2.61 \mathrm{E}+08$ & $2.40 \mathrm{E}+08$ & $2.61 \mathrm{E}+08$ & $2.00 \mathrm{E}+08$ \\
\hline adaptec4 & $2.14 \mathrm{E}+08$ & $2.53 \mathrm{E}+08$ & $2.09 \mathrm{E}+08$ & $2.52 \mathrm{E}+08$ & $1.80 \mathrm{E}+08$ \\
\hline bigblue1 & $1.10 \mathrm{E}+08$ & $5.00 \mathrm{E}+08$ & $1.10 \mathrm{E}+08$ & $4.98 \mathrm{E}+08$ & $9.40 \mathrm{E}+07$ \\
\hline bigblue2 & $1.65 \mathrm{E}+08$ & $1.79 \mathrm{E}+08$ & $1.64 \mathrm{E}+08$ & $1.78 \mathrm{E}+08$ & $1.30 \mathrm{E}+08$ \\
\hline bigblue3 & $4.15 \mathrm{E}+08$ & $5.86 \mathrm{E}+08$ & $4.67 \mathrm{E}+08$ & $5.85 \mathrm{E}+08$ & $3.60 \mathrm{E}+08$ \\
\hline bigblue4 & $8.97 \mathrm{E}+08$ & $1.04 \mathrm{E}+09$ & $9.09 \mathrm{E}+08$ & $1.03 \mathrm{E}+09$ & $7.60 \mathrm{E}+08$ \\
\hline AVG. & 1.18 & 1.92 & 1.19 & 1.92 & 1 \\
\hline
\end{tabular}

On legalized NTUplace3 [5] global placements on ISPD 2006 placement contest benchmarks, the HPWL is $1.44 \mathrm{X}, 1.25 \mathrm{X}$ and $1.43 \mathrm{X}$ of our legalizer compared to Tetris 17, Abacus [19, and Kahng 9], respectively. Compare the HPWL after our legalizer with the original HPWL of NTUplace3 global placement results [5], we can further reduce HPWL by $19 \%$. The details of HPWL experiments on ISPD 2006 benchmarks are listed in Table 4.

Table 4. Wirelength comparison between NTUplace3, Tetris, Abacus, Kahng, and ours on ISPD 2006 benchmarks

\begin{tabular}{|c|c|c|c|c|c|}
\hline ISPD 2006 Benchmarks & NTUplace3 [5] & Tetris [7] & Abacus [19] & Kahng [9] & Ours \\
\hline Name & HPWL & HPWL & HPWL & HPWL & HPWL \\
\hline adaptec5 & $4.01 \mathrm{E}+08$ & $4.95 \mathrm{E}+08$ & $4.07 \mathrm{E}+08$ & $4.94 \mathrm{E}+08$ & $3.14 \mathrm{E}+08$ \\
\hline newblue1 & $6.42 \mathrm{E}+07$ & $1.03 \mathrm{E}+08$ & $6.40 \mathrm{E}+07$ & $1.03 \mathrm{E}+08$ & $5.74 \mathrm{E}+07$ \\
\hline newblue2 & $2.16 \mathrm{E}+08$ & $2.46 \mathrm{E}+08$ & $2.20 \mathrm{E}+08$ & $2.45 \mathrm{E}+08$ & $2.02 \mathrm{E}+08$ \\
\hline newblue4 & $2.89 \mathrm{E}+08$ & $3.20 \mathrm{E}+08$ & $2.84 \mathrm{E}+08$ & $3.19 \mathrm{E}+08$ & $2.32 \mathrm{E}+08$ \\
\hline newblue5 & $5.06 \mathrm{E}+08$ & $5.34 \mathrm{E}+08$ & $5.01 \mathrm{E}+08$ & $5.32 \mathrm{E}+08$ & $4.23 \mathrm{E}+08$ \\
\hline newblue6 & $5.09 \mathrm{E}+08$ & $6.15 \mathrm{E}+08$ & $6.18 \mathrm{E}+08$ & $6.15 \mathrm{E}+08$ & $4.25 \mathrm{E}+08$ \\
\hline newblue7 & $1.13 \mathrm{E}+09$ & $1.30 \mathrm{E}+09$ & $1.25 \mathrm{E}+09$ & $1.30 \mathrm{E}+09$ & $9.24 \mathrm{E}+08$ \\
\hline AVG. & 1.19 & 1.44 & 1.25 & 1.43 & 1 \\
\hline
\end{tabular}

\subsection{Displacement}

In the second experiment, we report the total and maximum displacement on both ISPD benchmarks. On legalized NTUplace3 global placements on ISPD 2005 placement contest benchmarks, the total displacement is $25.76 \mathrm{X}, 1.09 \mathrm{X}$, and $27.16 \mathrm{X}$, the maximum displacement is $1.88 \mathrm{X}, 1.04 \mathrm{X}$, and $2.40 \mathrm{X}$ of our legalizer compared to Tetris [7, Abacus 19], and Kahng 9], respectively. The details of displacement experiments on ISPD 2005 benchmarks are listed in Table 5. 
Table 5. Displacement comparison between NTUplace3, Tetris, Abacus, Kahng, and ours on ISPD 2005 benchmarks

\begin{tabular}{|c|c|c|c|c|c|c|c|c|}
\hline ISPD 2005 Benchmarks & \multicolumn{2}{|c|}{ Tetris [7] } & \multicolumn{2}{c|}{ Abacus [19] } & \multicolumn{2}{c|}{ Kahng [9] } & \multicolumn{2}{c|}{ Ours } \\
\hline Name & TOTAL & MAX & TOTAL & MAX & TOTAL & MAX & TOTAL & MAX \\
\hline adaptec1 & $7.91 \mathrm{E}+07$ & 2490 & $5.89 \mathrm{E}+06$ & 1247 & $8.10 \mathrm{E}+07$ & 2490 & $6.00 \mathrm{E}+06$ & 1211 \\
\hline adaptec2 & $1.31 \mathrm{E}+08$ & 3432 & $1.90 \mathrm{E}+07$ & 3485 & $1.32 \mathrm{E}+08$ & 3432 & $1.90 \mathrm{E}+07$ & 3239 \\
\hline adaptec3 & $1.22 \mathrm{E}+08$ & 4581 & $5.11 \mathrm{E}+07$ & 5419 & $1.23 \mathrm{E}+08$ & 4577 & $4.80 \mathrm{E}+07$ & 5733 \\
\hline adaptec4 & $1.56 \mathrm{E}+08$ & 2197 & $2.74 \mathrm{E}+07$ & 1702 & $1.58 \mathrm{E}+08$ & 3161 & $2.60 \mathrm{E}+07$ & 1736 \\
\hline bigblue1 & $7.38 \mathrm{E}+08$ & 9319 & $4.63 \mathrm{E}+06$ & 1304 & $7.90 \mathrm{E}+08$ & 9319 & $4.80 \mathrm{E}+06$ & 1342 \\
\hline bigblue2 & $6.25 \mathrm{E}+07$ & 1143 & $1.31 \mathrm{E}+07$ & 871 & $6.77 \mathrm{E}+07$ & 3757 & $9.20 \mathrm{E}+06$ & 718 \\
\hline bigblue3 & $8.01 \mathrm{E}+08$ & 5886 & $4.20 \mathrm{E}+08$ & 6729 & $8.02 \mathrm{E}+08$ & 5886 & $3.50 \mathrm{E}+08$ & 6756 \\
\hline bigblue4 & $8.21 \mathrm{E}+08$ & 2115 & $6.56 \mathrm{E}+07$ & 5701 & $8.36 \mathrm{E}+08$ & 2115 & $6.00 \mathrm{E}+07$ & 5203 \\
\hline AVG. & 25.76 & 1.88 & 1.09 & 1.04 & 27.16 & 2.40 & 1 & 1 \\
\hline
\end{tabular}

On legalized NTUplace3 global placements on ISPD 2006 placement contest benchmarks, the total displacement is $5.27 \mathrm{X}, 1.15 \mathrm{X}$, and $5.43 \mathrm{X}$, the maximum displacement is $1.51 \mathrm{X}, 1.35 \mathrm{X}$, and $1.51 \mathrm{X}$ of our legalizer compared to Tetris [7, Abacus [19], and Kahng [9], respectively. The details of displacement experiments on ISPD 2006 benchmarks are listed in Table 6. It should be noted that Abacus [19] achieved the best published results in displacement but our legalizer still get better results due to the exact linear wirelength model.

Table 6. Displacement comparison between NTUplace3, Tetris, Abacus, Kahng, and ours on ISPD 2006 benchmarks

\begin{tabular}{|c|c|c|c|c|c|c|c|c|}
\hline ISPD 2006 Benchmarks & \multicolumn{2}{|c|}{ Tetris [7] } & \multicolumn{2}{c|}{ Abacus [19] } & \multicolumn{2}{c|}{ Kahng [9] } & \multicolumn{2}{c|}{ Ours } \\
\hline Name & TOTAL & MAX & TOTAL & MAX & TOTAL & MAX & TOTAL & MAX \\
\hline adaptec5 & $4.65 \mathrm{E}+08$ & 5117 & $9.12 \mathrm{E}+07$ & 3617 & $4.68 \mathrm{E}+08$ & 5117 & $8.79 \mathrm{E}+07$ & 4920 \\
\hline newblue1 & $1.44 \mathrm{E}+08$ & 3639 & $1.37 \mathrm{E}+07$ & 2990 & $1.45 \mathrm{E}+08$ & 3639 & $1.31 \mathrm{E}+07$ & 1459 \\
\hline newblue2 & $2.17 \mathrm{E}+08$ & 6162 & $1.05 \mathrm{E}+08$ & 4535 & $2.18 \mathrm{E}+08$ & 6162 & $7.37 \mathrm{E}+07$ & 2131 \\
\hline newblue4 & $2.17 \mathrm{E}+08$ & 2214 & $4.24 \mathrm{E}+07$ & 2808 & $2.30 \mathrm{E}+08$ & 2214 & $4.16 \mathrm{E}+07$ & 2327 \\
\hline newblue5 & $1.55 \mathrm{E}+08$ & 4030 & $3.52 \mathrm{E}+07$ & 1527 & $1.61 \mathrm{E}+08$ & 4030 & $3.31 \mathrm{E}+07$ & 1777 \\
\hline newblue6 & $8.97 \mathrm{E}+08$ & 2609 & $2.68 \mathrm{E}+08$ & 7485 & $9.07 \mathrm{E}+08$ & 2609 & $2.23 \mathrm{E}+08$ & 7514 \\
\hline newblue7 & $1.22 \mathrm{E}+09$ & 4248 & $3.99 \mathrm{E}+08$ & 10527 & $1.34 \mathrm{E}+09$ & 4248 & $3.20 \mathrm{E}+08$ & 7246 \\
\hline AVG. & 5.27 & 1.51 & 1.15 & 1.35 & 5.43 & 1.51 & 1.00 & 1.00 \\
\hline
\end{tabular}

\subsection{Running Time}

In the third experiment, we evaluate the running time on both ISPD benchmarks. On legalized NTUplace3 global placements on ISPD 2005 placement contest benchmarks, the running time is $5.79 \mathrm{X}, 36.18 \mathrm{X}$, and $5.94 \mathrm{X}$ of our legalizer compared to Tetris [7], Abacus [19], and Kahng [9], respectively. The details of running time experiments on ISPD 2005 benchmarks are listed in Table 7.

On legalized NTUplace3 global placements on ISPD 2006 placement contest benchmarks, the running time is $6.28 \mathrm{X}, 26.15 \mathrm{X}$, and $6.49 \mathrm{X}$ of our legalizer compared to Tetris [7, Abacus [19], and Kahng [9], respectively. The details of 
running time experiments on ISPD 2006 benchmarks are listed in Table 8. It should be noted that Tetris [7] achieved the fastest published results but our legalizer still get better results due to the row indexing and fast trial placement techniques.

Table 7. Runtime comparison between NTUplace3, Tetris, Abacus, Kahng, and ours on ISPD 2005 benchmarks

\begin{tabular}{|c|c|c|c|c|}
\hline ISPD 2005 Benchmarks & Tetris [7] & Abacus [19] & Kahng [9] & Ours \\
\hline Name & TIME (s) & TIME (s) & TIME (s) & TIME (s) \\
\hline adaptec1 & 32 & 101 & 34 & 16 \\
\hline adaptec2 & 44 & 321 & 47 & 11 \\
\hline adaptec3 & 163 & 647 & 169 & 24 \\
\hline adaptec4 & 213 & 682 & 218 & 47 \\
\hline bigblue1 & 38 & 110 & 40 & 18 \\
\hline bigblue2 & 360 & 601 & 365 & 72 \\
\hline bigblue3 & 1453 & 15033 & 1465 & 82 \\
\hline bigblue4 & 1272 & 4456 & 1304 & 304 \\
\hline AVG. & 5.79 & 36.18 & 5.94 & 1 \\
\hline
\end{tabular}

Table 8. Runtime comparison between NTUplace3, Tetris, Abacus, Kahng, and ours on ISPD 2006 benchmarks

\begin{tabular}{|c|c|c|c|c|}
\hline ISPD 2006 Benchmarks & Tetris [7] & Abacus [19] & Kahng [9] & Ours \\
\hline Name & TIME (s) & TIME (s) & TIME (s) & TIME (s) \\
\hline adaptec5 & 304 & 2225 & 312 & 51 \\
\hline newblue1 & 32 & 123 & 35 & 24 \\
\hline newblue2 & 389 & 1032 & 395 & 53 \\
\hline newblue4 & 274 & 1327 & 281 & 49 \\
\hline newblue5 & 598 & 1869 & 612 & 116 \\
\hline newblue6 & 839 & 4917 & 854 & 188 \\
\hline newblue7 & 5163 & 16844 & 5245 & 365 \\
\hline AVG. & 6.28 & 26.15 & 6.40 & 1 \\
\hline
\end{tabular}

Figure 14 (a) shows the global placement result of "adaptec1" obtained from NTUplace3. To further reveal the difference of Tetris, Abacus, Kahng, and ours, we plot the movement pictures during legalization in Figure 14 (b), (c), (d), and (e). 


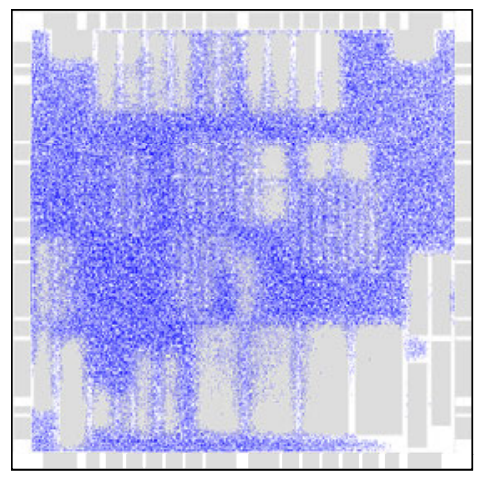

(a)

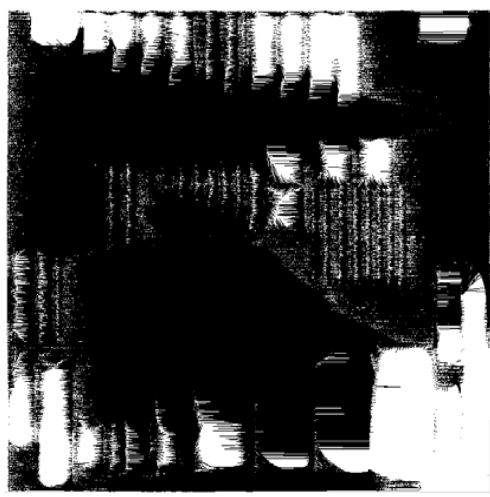

(b)

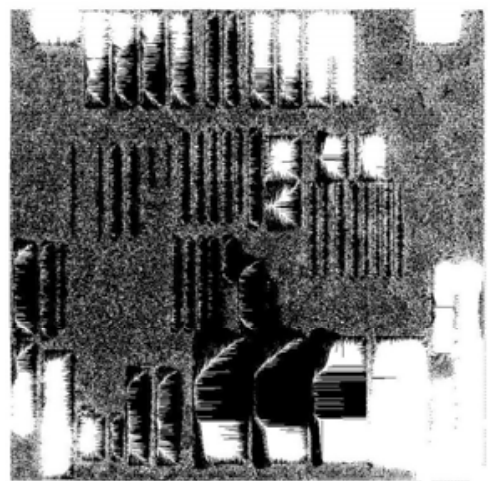

(d)

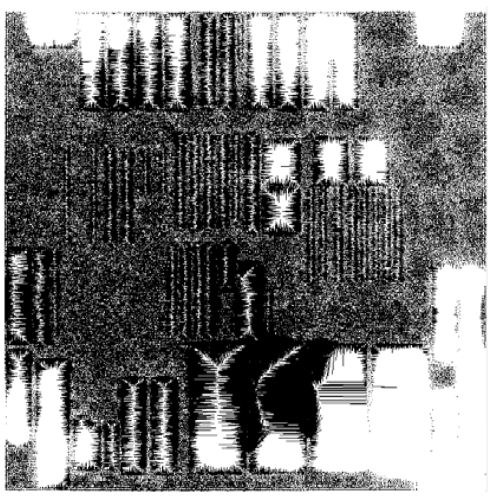

(c)

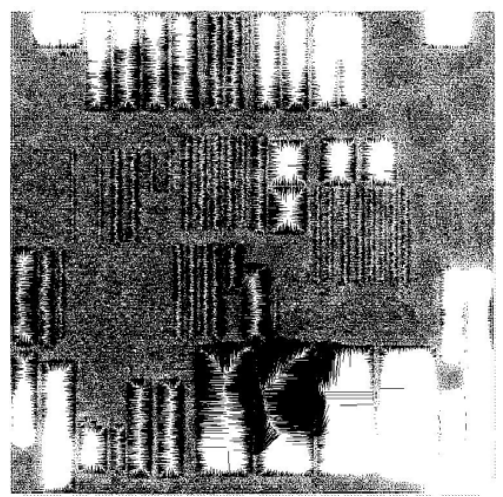

(e)

Fig. 14. (a) Global placement result of adaptec1. (b) Cell movement during Tetris [7]. (c) Cell movement during Abacus [19]. (d) Cell movement during Kahng [9]. (e) Ours. 


\section{Conclusions}

In this paper, we proposed a fast legalization algorithm with simultaneous displacement and HPWL minimization. The main contributions of our work are: (1) a fast row selection technique by using k-medoid clustering approach; (2) an exact linear wirelength model to minimize both wirelength and total displacement; (3) a constant time approach to determine the median in trial placement stage. Compared with the state-of-the-art algorithms, experimental results have shown that our legalizer obtains very high-quality results on legalized NTUplace3 global placements on both ISPD 2005 and 2006 placement contest benchmarks.

\section{References}

1. Agnihorti, A., Yildiz, M.C., Khatkhate, A., Mathur, A., Ono, S., Madden, P.H.: Fractional cut: Improved recursive bisection placement. In: Proc. ICCAD, pp. 307-310 (2003)

2. Brenner, U., Vygen, J.: Legalizing a placement with minimum total movement. IEEE TCAD 23(12), 1597-1613 (2004)

3. Brenner, U., Struzyna, M.: Faster and better global placement by a new transportation algorithm. In: Proc. DAC, pp. 591-596 (2005)

4. Chan, T., Cong, J., Sze, K., Xie, M.: mPL6: Enhanced multilevel mixed-size placement. In: Proc. ISPD, pp. 212-214 (2006)

5. Chen, T.-C., Jiang, Z.-W., Hsu, T.-C., Chen, H.-C., Chang, Y.-W.: NTUplace3: A high-quality mixed-size analytical placer considering preplaced blocks and density constraints. In: Proc. ICCAD, pp. 187-192 (2006)

6. Doll, K., Johannes, F.M., Antreich, K.J.: Iterative placement improvement by network flow methods. IEEE TCAD 13(10), 1189-1200 (1994)

7. Hill, D.: Method and system for high speed detailed placement of cells within integrated circuit designs. U.S. Patent 6370673 (April 2002)

8. Hur, S.-W., Lillis, J.: Mongrel: Hybrid techniques for standard cell placement. In: Proc. ICCAD, pp. 165-170 (2000)

9. Kahng, A.B., Tucker, P., Zelikovsky, A.: Optimization of Linear Placements for Wirelength Minimization with Free Sites. In: Proc. ASP-DAC 1999, pp. 241-244 (1999)

10. Kahng, A.B., Markov, I.L., Reda, S.: On legalization of row-based placements. In: Proc. GLS-VLSI, pp. 214-219 (2004)

11. Kahng, A.B., Wang, Q.: Implementation and extensibility of an analytic placer. IEEE TCAD 24(05), 734-747 (2005)

12. Lucasius, C.B., Dane, A.D., Kateman, G.: On k-medoid clustering of large data sets with the aid of a genetic algorithm: background, feasibility and comparison. Analytica Chimica Acta 282(3), 647-669 (1993)

13. Luo, T., Ren, H., Alpert, C.J., Pan, D.Z.: Computational geometry based placement migration. In: Proc. DAC, pp. 41-47 (2007)

14. Pan, M., Viswanathan, N., Chu, C.: An efficient and effective detailed placement algorithm. In: Proc. ICCAD, pp. 48-55 (2005)

15. Ren, H., Pan, D.Z., Alpert, C.J., Villarrubia, P.: Diffusion-based placement migration. In: Proc. DAC, pp. 515-520 (2005) 
16. Roy, J.A., Papa, D.A., Adya, S.N., Chan, H.H., Ng, A.N., Lu, J.F., Markov, I.L.: Capo: Robust and scalable open-source min-cut floorplacer. In: Proc. ISPD, pp. 224-226 (2005)

17. Sarrafzadeh, M., Wang, M.: NRG: Global and detailed placement. In: Proc. ICCAD, pp. 532-537 (1997)

18. Spindler, P., Johannes, F.M.: Fast and robust quadratic placement based on an accurate linear net model. In: Proc. ICCAD, pp. 179-186 (2006)

19. Spindler, P., Schlichtmann, U., Johannes, F.M.: Abacus: Fast legalization of standard cell circuits with minimal movement. In: Proc. ISPD, pp. 47-53 (2008)

20. Wang, T.C., Wong, D.F.: Optimal floorplan area optimization. IEEE TCAD 11, 992-1002 (1992)

21. Wong, D.F., Liu, C.L.: A new algorithm for floorplan design. In: Proc. DAC, pp. 101-107 (1986)

22. http://www.ispd.cc/ 